\title{
Conduction mechanism in codeposited AgSe thin films
}

\author{
P S NIKAM and H S AHER \\ P G Department of Physical Chemistry, M S G College, Malegaon Camp 423 105, India \\ MS received 26 April 1993; revisad 26 October 1993
}

\begin{abstract}
Thin films of AgSe of varying compositions and thicknesses have been formed on glass substrates employing the three-lemperature method. $I-V$ characteristics and thermo-

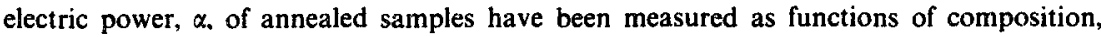
thickness and temperature of the films. Films exhibit $n$-type conductivity. Nonohmic conduction in films of $\mathrm{Ag}_{x} \mathrm{Se}_{1-x}(0<x<0.5)$ and $\mathrm{Ag}_{x} \mathrm{Se}_{1-x}(0>x>0.5)$ have been accounted for on the basis of the theory of Rose of defect insulator containing shallow traps and on Schottky emission respectively.
\end{abstract}

Keywords. Thermoelectric power; $I-V$ characteristics; space charge limited current; PooleFrenkel; Richardson Schottky emission.

\section{Introduction}

Silver selenide is a good thermoelectric material in bulk solid state. A survey of the literature (Chouching-Liang and Pinsker 1962; Abdullayev et al 1980, 1983; Tomoyose 1980; Constantinescu 1981, 1983; Ramzan Zade et al 1981; Saito et al 1981; Bernede and Bouchairi 1983; Honing and Thomas 1987; Damodara Das and Karunakaran 1989; Sharma et al 1990) shows that there are few studies of electrical properties of AgSe system in bulk as well as in thin states over the entire range of composition. Since the chemical composition of the deposits affects the transport properties critically, we have studied in detail transport and galvanomagnetic properties as affected by the concentration of the two components (AgSe) here.

\section{Experimental}

Thin films of AgSe (99.999\%) were prepared by vacuum evaporation method (Nikam and Aher 1993). Silver was evaporated directly from a preflashed conical basket of tungsten wire and selenium powder from conical mica crucibles with nichrome wire windings. The evaporation was done at room temperature in an IBP TORR-120 coating unit, under a vacuum of the order of $10^{-5}$ torr. The flux rate from each source could easily be adjusted by controlling the current through each filament. Both elements were simultaneously heated. After adjusting the flux rates from the two sources by varying the source current, films of varying compositions were obtained. They were annealed at $\sim 100^{\circ} \mathrm{C}$ for $8 \mathrm{~h}$ in vacuum.

The methods employed to determine composition, thickness and uniformity of the film were similar to those reported earlier (Nikam and Pawar 1990, 1991a, b). The composition of the films was determined by employing absorptiometric spectroscopy at $620 \mathrm{~nm}$ (Charlot 1964). To check the uniformity of the film as regards its composition, different portions of the film were subjected to absorptiometric spectroscopic analysis. The analysis confirmed that the films were of uniform composition and thickness. 
Film thickness was measured using multiple beam interferometry. Film thickness $(d)$ has also been measured by gravimetric (Nikam and Aher 1993) method:

$$
d=\frac{M}{g \times A} \mathrm{~cm},
$$

where $A$ is surface area of the film, $M$ the mass of the film, and $g$ the density of the film material expressed as

$$
g=x_{1} g_{1}+x_{2} g_{2}
$$

where $g_{1}, g_{2}$ and $x_{1}, x_{2}$ are densities and atomic fractions of $\mathrm{Ag}$ and Se elements respectively. Films prepared for Seebeck effect and $I-V$ characteristic measurements had composition ranging between $\mathrm{Ag}_{10} \mathrm{Se}_{90}$ and $\mathrm{Ag}_{80} \mathrm{Se}_{20}$ and thickness between 1200 and $5000 \AA$. The thermoelectric power $(\alpha)$ was measured using the integral method in vacuum by pressure contacts (Nikam and Pawar 1985, 1990, 1991a, b). The distance between hot and cold ends was $\sim 3 \mathrm{~cm}$. Thermal emfs were measured by means of a microvolt potentiometer (Ajco) connected to a sensitive spot galvanometer (Ajco, p-42).

For $I-V$ measurements, films of AgSe of different thicknesses and compositions were grown over Al base electrodes on clean glass substrates held at $300 \mathrm{~K}$ through suitable masks in a dynamic pressure of $10^{-5}$ torr. Aluminium counterelectrodes were vacuumdeposited over the AgSe films to complete the sandwiched structure.

The $I-V$ characteristics were measured using a DC microvoltmeter (Ajco model 79016), a vacuum tube voltmeter (Ruttonsha Simpson model 321 1), a standard resistance, etc.

\section{Results}

Thermoelectric power $(\alpha)$ of all $\mathrm{Ag}-\mathrm{Se}$ deposits was measured using the integral method. All values of thermoelectric power were negative indicating that electrons were the majority carriers, which is in agreement with the results reported earlier (Kienel 1960). Figure 1 shows the plot of thermal emf as a function of temperature

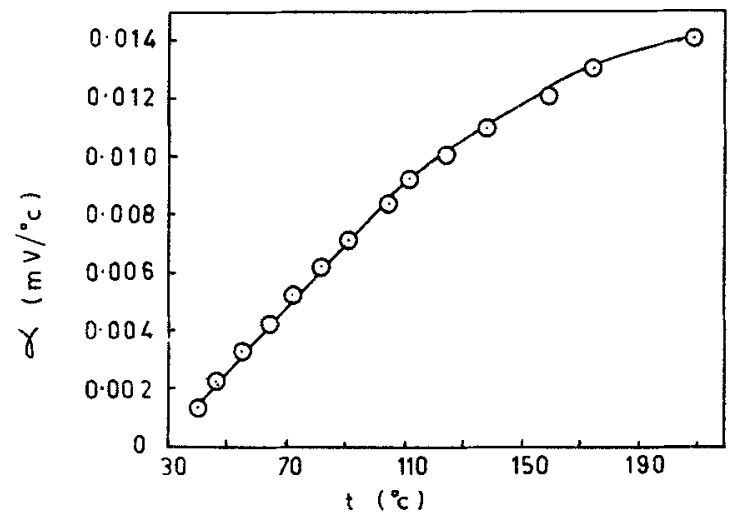

Figure 1. Variation of thermal emf as a function of temperature difference for $\mathrm{Ag}_{x} \mathrm{Se}_{1-x}(0<x<0 \cdot 5)$ films of thickness $3300 \AA$. 


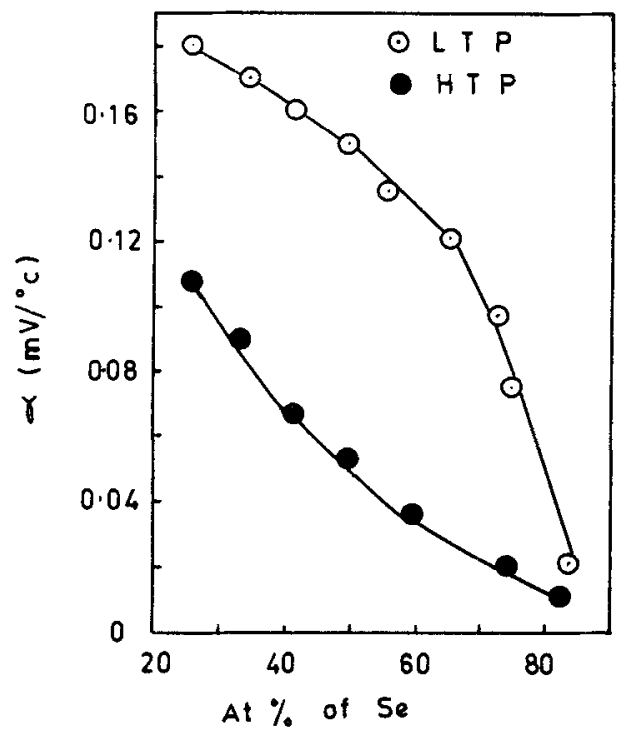

Figure 2. Variation of TEP $(x)$ with composition of the film of thickness $3200 \AA$.

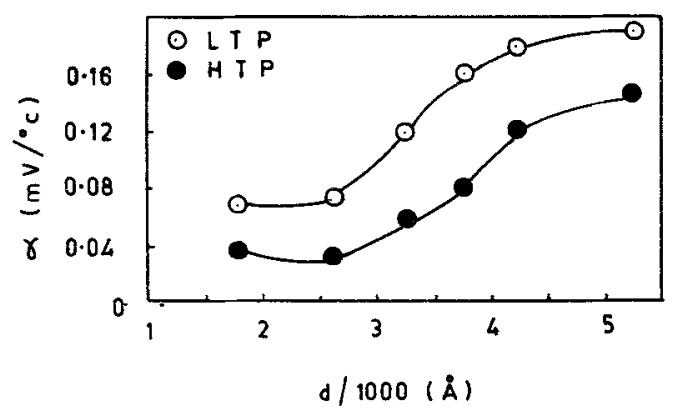

Figure 3. TEP as a function of film thickness.

difference between the hot and cold ends during heating for $\operatorname{Ag}_{x} \operatorname{Se}_{1-x}(0<x<0.5)$ films of thickness $3300 \AA$ in the temperature range $300-500 \mathrm{~K}$. It is seen from the figure that thermal emf increases nonlinearly with increase in temperature difference up to a phase transition (semiconducting to metallic) temperature of $130^{\circ} \mathrm{C}$ (Nikam and Aher 1993), and then it increases slowly.

Figure 2 illustrates the variation of TEP $(\alpha)$ with composition of the film of thickness $3200 \AA$. The low-temperature phase (LTP) which is semiconducting has higher TEP value than the high-temperature metallic phase (HTP). In both the phases, TEP decreases with increase of $\mathrm{Se}$ in the film, probably due to decrease of free electron density in the film with addition of semiconducting Se. To analyse dimensional effects, the thermoelectric power in both phases is plotted as a function of film thickness $\left(\mathrm{Ag}_{35} \mathrm{Se}_{65}\right)$. Figure 3 shows these plots. It is seen that the TEP of the thinnest film is the lowest (in magnitude) in low- as well as high-temperature phases, and it increases 


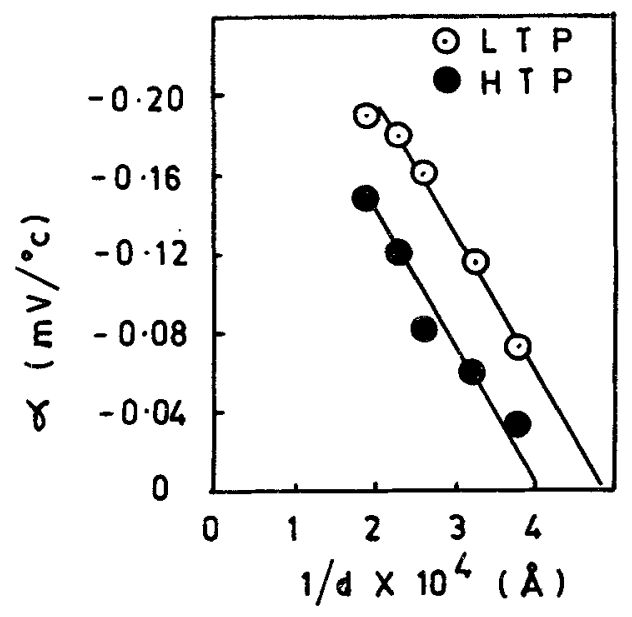

Figure 4. TEP against reciprocal thickness of the film having composition $\mathrm{Ag}_{35} \mathrm{Se}_{65}$.

(in magnitude) rapidly with increasing thickness up to about $2500 \AA$ and thereafter increases slowly with a further increase in thickness, attaining saturation.

Figure 4 shows plots of thermoelectric power against reciprocal thickness of the film having composition $\mathrm{Ag}_{35} \mathrm{Se}_{65}$ in low- as well as high-temperature regions. It is seen that both the curves are linear, the plots become more scattered at lower thicknesses, indicating an inverse relationship between $\alpha_{F}$ (TEP of film) and $d$. The intercept on the $y$ axis gives the bulk thermoelectric power $\alpha_{B}$ or grain-boundary thermoelectric power $\alpha_{g} . \alpha_{B}$ is the thermoelectric power of the bulk sample of very large grain size, nearly equal to several micrometres, where the grain-boundary scattering effects are insignificant, while $\alpha_{g}$ is defined as the thermoelectric power of a bulk sample having the same grain size as the films to that the grain-boundary scattering effects are significant. It is interesting to note that the slopes of the linear plots of $\alpha_{F}$ vs $1 / d$ are nearly the same, proving independence of the constant of proportionality between $\alpha_{F}$ and $1 / d$. The plot at lower temperature is above that at higher temperature. This is to be expected because of the decrease of thermoelectric power with increase of temperature of the films.

In the free-electron approximation and for a spherical Fermi surface, the thermoelectric power of a metal and/or degenerate semiconductor is given by (Ziman 1962)

$$
\alpha_{B}=\frac{\pi^{2} k_{B}^{2} T}{3 e E_{F}}(U+V) .
$$

In the present investigation $\alpha_{B}$ decreases with the rise in temperature suggesting that the material behaves as a non-degenerate semiconductor (Conn and Taylor 1960).

According to the classical size-effect theory, the thermoelectric power $\alpha_{F}$ of a thin film of thickness $d$ is given by (Mayer 1955)

$$
\alpha_{F}=\alpha_{B}\left[1-\frac{3}{8}(1-P) \frac{U}{1+U} \frac{\lambda_{B}}{d}\right]
$$

The above expression is valid for monocrystaliine films as it does not take into account the contribution to the thermoelectric power due to grain-boundary 
scattering. Pichard et al (1980) derived an expression for the thermoelectric power of a polycrystalline thin film as

$$
x_{F}=\frac{\pi^{2} k_{B}^{2} T}{3 e E_{F}}\left[V+U \frac{\sigma_{g}}{\sigma_{B}}-\frac{3}{8}(1-P) \frac{\lambda_{B}}{d} U\left[\frac{\sigma_{g}}{\sigma_{B}}\right]^{2}\right],
$$

where $P$ is the specularity parameter, giving the fraction of the electrons specularly scattered from the surfaces, $\hat{\lambda}_{B}$ the mean free path, $U$ and $V$ are the rate of change of mean free path with the energy evaluated at the Fermi energy and the rate of variation of the Fermi surface area with energy evaluated at the Fermi energy respectively, and expressed as

and

$$
U=\left(d \ln \lambda_{B} / d \ln E\right)_{E=E_{F}},
$$

$$
V=(d \ln A / d \ln E)_{E=E_{F}} \text {. }
$$

In (3) and (5) $e$ is the charge (in magnitude and sign) of the charge carrier, $k_{B}$ the Boltzmann constant, and $T$ the temperature in $K, \sigma_{g}$ and $\sigma_{B}$ are the grain-boundary conductivity and the bulk conductivity respectively.

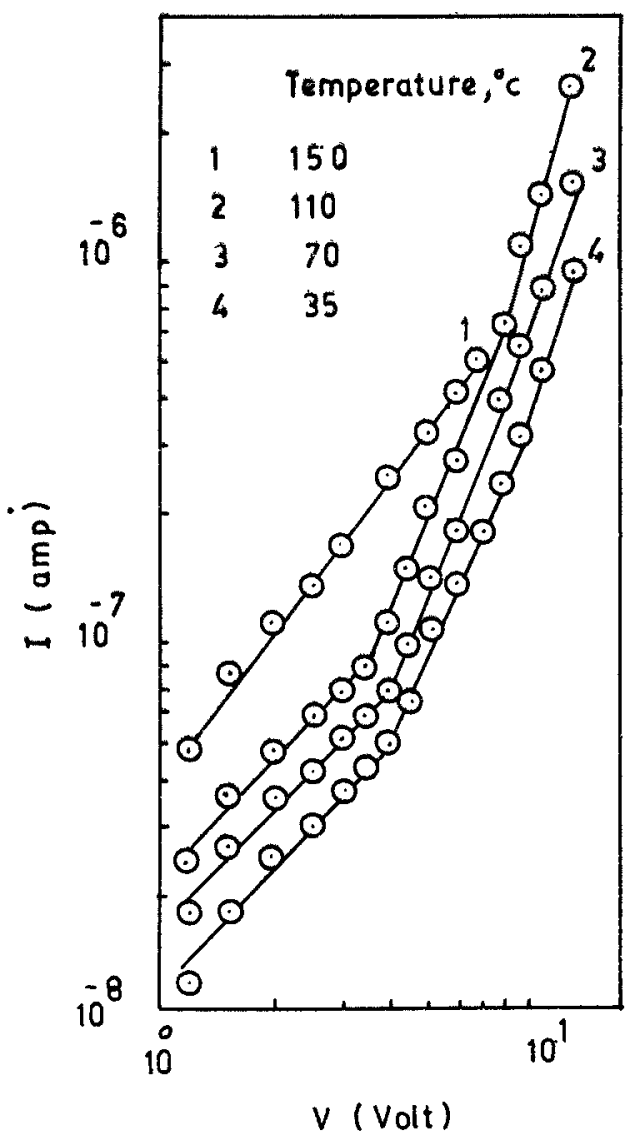

Figure 5. $I-V$ characteristics of $\operatorname{Ag}_{x} \mathrm{Se}_{1-x}(0<x<0 \cdot 5)$ films of thickness $2300 \AA$. 
According to (4) and (5), a plot of $\alpha_{F}$ vs $1 / d$ will be a straight line in either case. However, the intercept on the $y$ axis gives the bulk thermoelectric power, $\alpha_{B}$, or grainboundary thermoelectric power, $\alpha_{g}$, according to the above equations respectively. Thus the nature of the two equations is the same except for the difference in the constant parameters involved in the functional relationship.

The values of $x_{B}$ or $x_{g}$ in low-temperature $\left(25-130^{\circ} \mathrm{C}\right)$ and high-temperature $\left(>130^{\circ} \mathrm{C}\right)$ regions obtained from the intercept of the $x_{F}$ vs $1 / d$ plots (figure 4) are $-340 \mu \mathrm{V} /{ }^{\circ} \mathrm{C}$ and $-280 \mu \mathrm{V} /{ }^{\circ} \mathrm{C}$ respectively. Since values of $\alpha_{B}$ or $\alpha_{g}$ for AgSe system over the entire composition range are unavailable in the literature comparison is not possible.

Figure 5 shows the $I-V$ characteristics of $\mathrm{Ag}_{x} \mathrm{Se}_{1-x}(0<x<0.5)$ films of thickness $2300 \AA$ at different temperatures. It is seen from these curves that for temperatures $\leqslant 150^{\circ} \mathrm{C}$, three regions of conduction, i.e. ohmic conduction with slope of curves $\sim 1$ at low field, SCLC conduction with slope $\sim 2$ at intermediate fields, and emissionlimited conduction with slope $\geqslant 3$ at high fields, are observed whereas at temperatures $>150^{\circ} \mathrm{C}$ only the ohmic behaviour is observed.

Figure 6 shows the $I-V$ characteristics of $\mathrm{Ag}_{x} \mathrm{Se}_{1-x}(0<x<0.5)$ films at a constant temperature, $\sim 303 \mathrm{~K}$, but for different thicknesses. It is observed from these curves

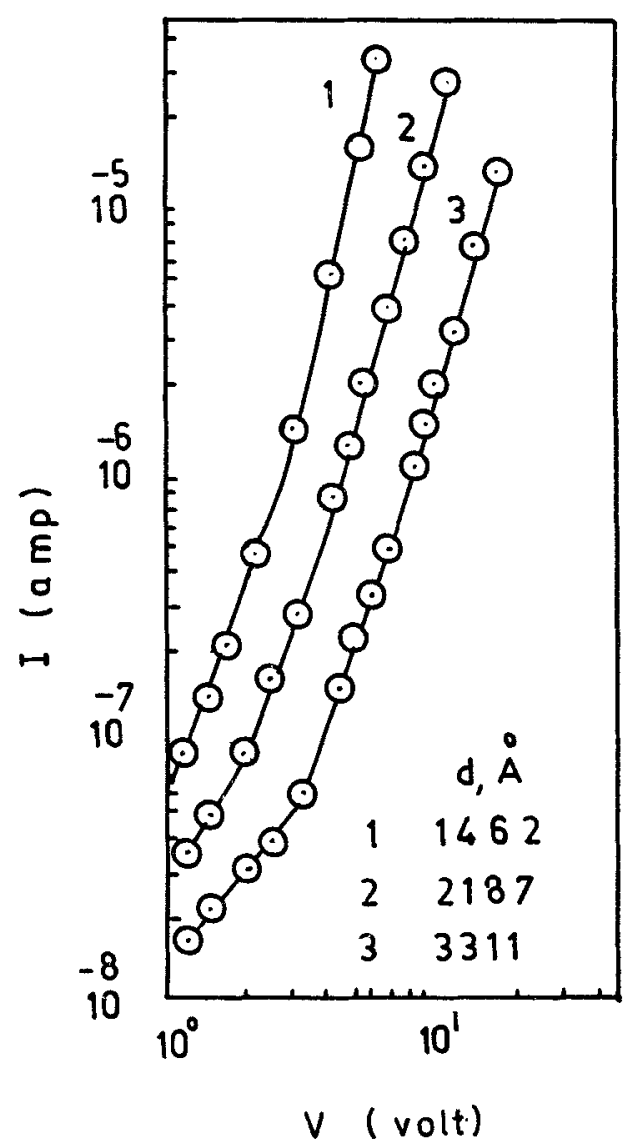

Figure 6. $I-V$ characteristics of $\mathrm{Ag}_{x} \mathrm{Se}_{1-x}(0<x<0.5)$ films at constant temperature. 


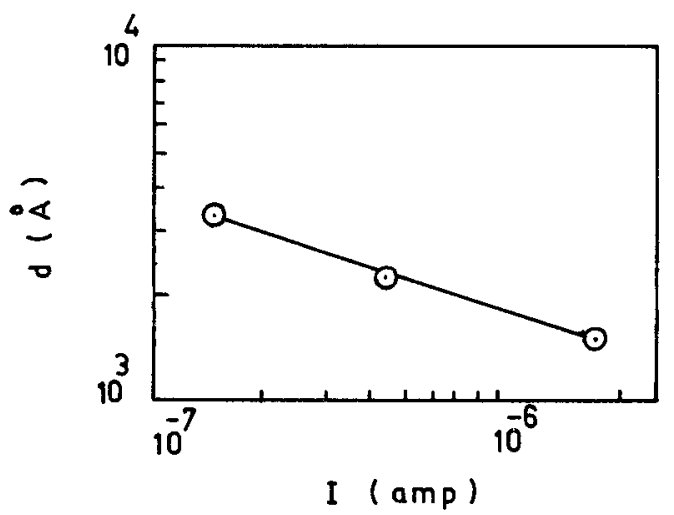

Figure 7. $\log -\log$ plots of $I$ vs $d$ for $\mathrm{Ag}_{x} \mathrm{Se}_{1-x}(0<x<0-5)$ films.

that the transition voltage, $V_{\text {trans }}$, separating ohmic and SCL conduction regions depends on the thickness $d$ of the films. Log- $\log$ plots of $V_{\text {trans }}$ vs thickness $d$ (results not shown) have shown a $V_{\text {trans }} \propto d^{2}$ dependence, which indicates that the intermediate field region is governed by space charge limited currents (SCLC). For SCL conduction it is known (Lamb 1967; Lamport and Mark 1970) that, in the presence of shallow traps, current density $I$ and $V_{\text {trans }}$ should obey

and

$$
I=(9 / 8) \mu_{c} \varepsilon V^{2} / d^{3}
$$

$$
V_{\text {trans }}=(8 / 9) e n_{t} d^{2} / \varepsilon
$$

where $e$ is the electronic charge, $V$ the applied voltage, $\varepsilon$ the high-frequency dielectric constant of the material, $\mu_{c}$ the effective drift mobility of charge carriers in the presence of shallow traps, and $n_{t}$ the trapped carrier density.

According to (8), for the current to be limited by SCLC in the $V^{2}$ region, $I \propto d^{-3}$. Using the data of figure $6, \log -\log$ plots of $I$ vs $d$ have been drawn (figure 7 ) and a slope $\sim-.3$ has been obtained suggesting that $I \propto d^{-3}$.

The activation energies of the charge carriers responsible for space charge formation have been evaluated by usual Arrhenius-type $\log I$ vs $T^{-1}$ plots (figure 8) and have been found to be $\sim 0.098 \mathrm{eV}$ at all fields. Such an observation suggests that perhaps the energy level of traps to which the charge carriers are injected from the electrodes does not vary with the applied field and the contact remains injecting all the time, which is the prerequisite (Lamb 1967) for observing the space charge limited currents.

Figure 9 shows the $I-V$ characteristics of $\mathrm{Ag}_{x} \mathrm{Se}_{1-x}(0>x>0.5)$ films of thickness $\sim 2900 \AA$. Similar plots (result not shown) have been obtained for different thicknesses of films with same composition. It is seen that each curve starts with ohmic region $(I \propto V)$ and then changes to nonohmic region $\left(I \propto V^{n}\right)$. In nonohmic region, the value of $n\left(I \propto V^{n}\right)$, which is more than one but less than 2, rules out the possibility of SCLC conduction in defect insulators containing exponential distribution of shallow traps. The nonlinear plots of $\log \left(I / V^{2}\right)$ vs $1 / V$ (not shown) suggests that $I-V$ characteristics in nonlinear region cannot be explained by a tunnel effect of Fowler-Nordheim type. The straight-line plots of $\log I$ vs $\sqrt{V}$ (figure 10) in high-field region suggest Schottky or Poole-Frenkel conduction mechanisms expressed by the relations (10) and (11) 
respectively;

and

$$
I=A T^{2} \exp \left[\left(\beta_{S} V^{1 / 2}-\varphi_{0}\right) / k_{B} T\right]
$$

where

$$
I=\beta \exp \left[\left(\beta_{P F} V^{1 / 2}-\varphi_{1}\right) / k_{B} T\right]
$$

$$
\beta_{P F}=2 \beta_{S}=2\left(e^{3} / 4 \pi \varepsilon_{0} \varepsilon_{d}\right)^{1 / 2}
$$

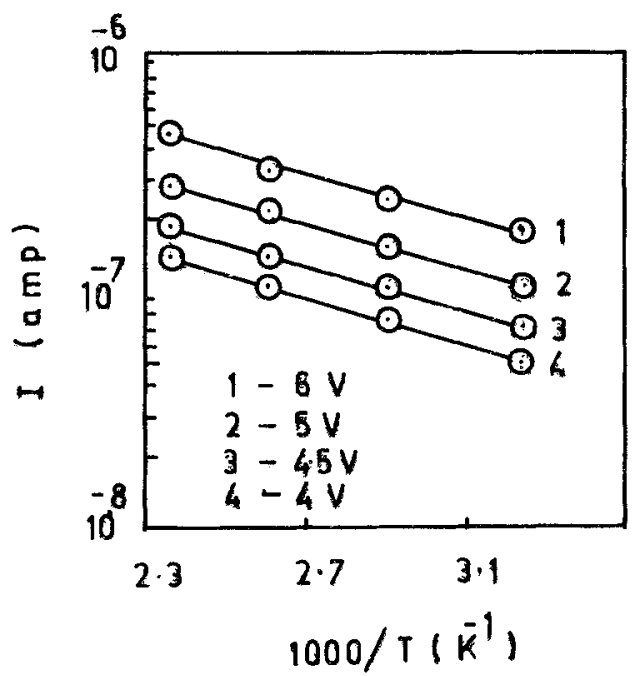

Figure 8. $\log I$ vs $T^{-1}$ for $\operatorname{Ag}_{x} 8 e_{1-x}(0<x<0.5)$ films.

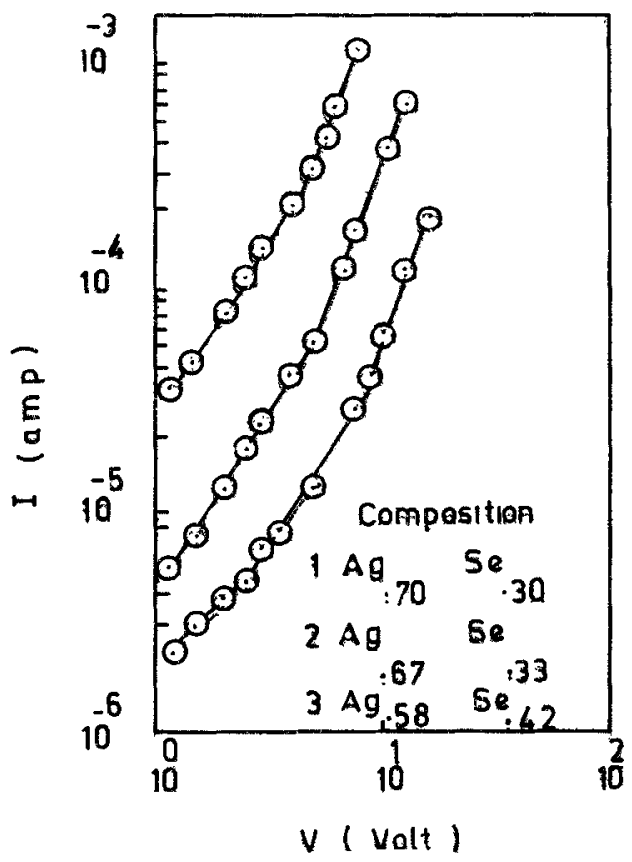

Figure 9. $I-V$ characteristics of $\mathrm{Ag}_{x} \mathrm{Se}_{1-x}(0>x>0 \cdot 5)$ films of thickness $\sim 2900 \AA$. 


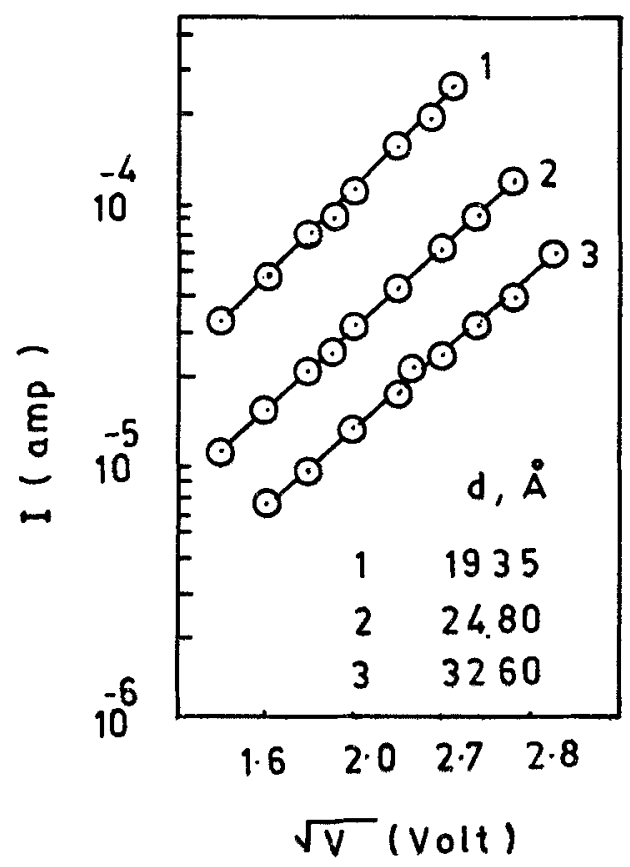

Figure 10. $\log I$ vs $\sqrt{V}$ for $\operatorname{Ag}_{x} \mathrm{Se}_{1-x}(0<x<0.5)$ films.

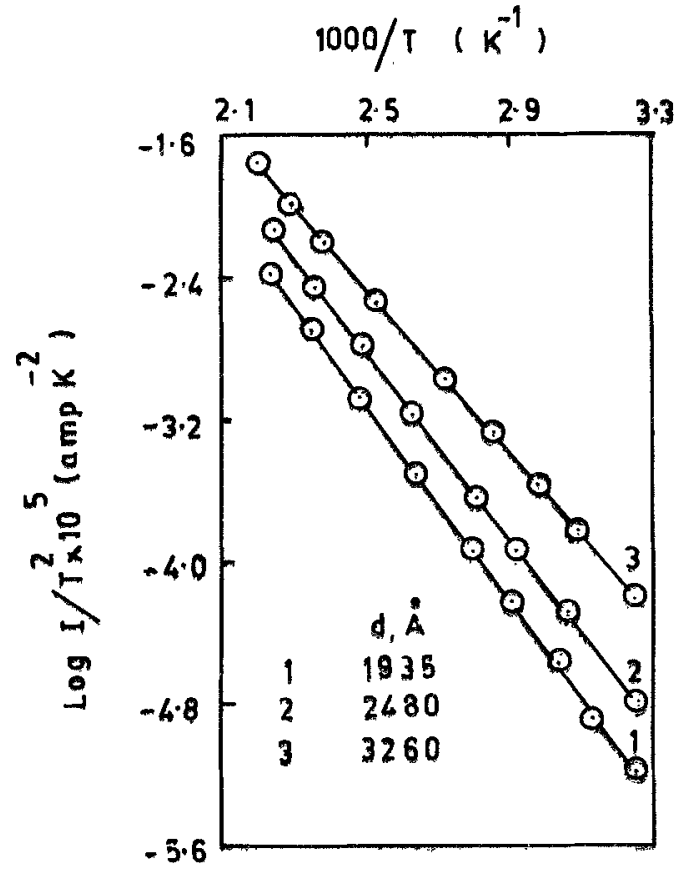

Figure 11. $\log l / T^{2}$ vs $10^{3} / T$ for different thickness of the film of $\operatorname{Ag}_{x} \operatorname{Se}_{1-x}(0>x>0 \cdot 5)$. 
Table 1. Variation of barrier height $(\varphi)$ of $\mathrm{Ag}_{x} \mathrm{Se}_{1-x}(0>x>0.5)$ films with thickness $(d)$.

\begin{tabular}{ll}
\hline$d(\AA)$ & $\varphi(\mathrm{eV})$ \\
\hline 1935 & 0.057 \\
2480 & 0.053 \\
3260 & 0.048 \\
\hline
\end{tabular}

The straight-line graphs (figure 11) between $\log I / T^{2}$ and $10^{3} / T$ for different thicknesses of the film of $\operatorname{Ag}_{x} \mathrm{Se}_{1-x}(0>x>0.5)$ corroborate that Schottky emission is responsible for nonohmic conduction in intermediate-voltage region. The slopes of the graphs in figure 11 give barrier heights (activation energy) which are included in table 1.

In high-voltage region, $n \geqslant 3$ suggests emission-limited conduction.

It is concluded from the above studies that thermoelectric power measurements of vacuum-evaporated layers of AgSe carried out in the temperature range $300-500 \mathrm{~K}$ show $n$-type conductivity irrespective of composition and thickness of the film. $I-V$ characteristic measurements show space charge limited conduction in $\mathrm{Ag}_{x} \mathrm{Se}_{1-x}$ $(0<x<0.5)$ films, and Richardson-Schottky emission in $\operatorname{Ag}_{x} \operatorname{Se}_{1-x}(0>x>0.5)$ films. The interstitial excess Se atoms are probably responsible for the effect in $\mathrm{Ag}_{x} \mathrm{Se}_{1-x}$ $(0<x<0.5)$ films.

\section{Acknowledgement}

The authors thank Mr S S Patil, Principal, M S G College, for laboratory facilities.

\section{References}

Abdullayev A G, Aliyev V K, Fufayeva T G and Skugareva L J 1980 Thin Solid Films 73 L-7 Abdullayev A G, Shafizade R B, Krupnikov E S and Kiriluk K V 1983 Thin Solid Films 106175 Bernede J C and Bouchairi B E 1983 C. R. Seances Acad. Sci. Ser. II 296173

Charlot G 1964 Colorimetric determination of elements (Amsterdam: Elsevier)

Chou Ching - Liang and Pinsker Z G 1962 Sov. Phys. Crystallogr. 752

Conn J B and Taylor R C 1960 J. Electrochem. Soc. 107977

Constantinescu L V 1981 Rev. Roum. Phys. 261123

Constantinescu L V 1983 Rev. Roum. Phys. 2873

Damodara Das V and Karunakaran D 1989 Phys. Rev. B39 10872

Honing V and Thomas A 1987 Phys. Status Solidi A100 K-81

Kienel G 1960 Ann. Physik Cep. 75229

Lamb D R 1967 Electrical conduction mechanism in thin insulating films (London: Methuen)

Lamport M A and Mark P 1970 Current injection in solids (New York: Academic Press) p. 21

Mayer H 1955 Phys. dunner Schichten (Stuttgart: Wissenschaftliche) 2

Nikam P S and Pawar R R 1985 Indian J. Pure Appl. Phys. 23171

Nikam P S and Pawar R R 1990 Bull. Mater. Sci. 13343

Nikam P S and Pawar R R 1991a Indian J. Pure Appl. Phys. 29263

Nikam P S and Pawar R R 1991b Pramana-J. Phys. 36629

Nikam P S and Aher H S 1993 Indian J. Pure Appl. Phys. 3179

Pichard C R, Tellier C R and Tosser A J 1980 J. Phys. F10 2009

Ramazan Zade M G, Aliev S A, Berdiera N A and Agaev A M 1981 Sov. Phys. J. 24919

Saito I, Sato M and Shiojiri M 1981 Thin Solid Films 79257

Sharma K C, Sharma R P and Garg J C 1990 Indian J. Pure Appl. Phys. 28546

Tomoyose T 1980 Bull. Coll. Sci. Uni. Ryukyas, Japan 301

Ziman J M 1962 Electrons and Phonons (Oxford: Clarendon) p. 397 